\title{
Live cell analysis at sea reveals divergent thermal performance between photosynthetic ocean microbial eukaryote populations
}

\author{
Allison Skinner Mclnnes ${ }^{1,2}$ - Olivier F. Laczka $\mathbb{1 D}^{1} \cdot$ Kirralee G. Baker ${ }^{1,3} \cdot$ Michaela E. Larsson $^{1}$ • \\ Charlotte M. Robinson ${ }^{1,4}$ - Jennifer S. Clark ${ }^{1,5} \cdot$ Leonardo Laiolo $^{1,6} \cdot$ Marco Alvarez $^{1} \cdot$ Bonnie Laverock $^{1,7}$. \\ Colin T. Kremer ${ }^{8}{ }^{8} \cdot$ Erik van Sebille $^{9,10} \cdot$ Martina A. Doblin (iD ${ }^{1}$
}

Received: 1 October 2018 / Revised: 31 December 2018 / Accepted: 14 January 2019 / Published online: 31 January 2019

(c) International Society for Microbial Ecology 2019

\begin{abstract}
Experimentation at sea provides insight into which traits of ocean microbes are linked to performance in situ. Here we show distinct patterns in thermal tolerance of microbial phototrophs from adjacent water masses sampled in the south-west Pacific Ocean, determined using a fluorescent marker for reactive oxygen species (ROS). ROS content of pico-eukaryotes was assessed after 1,5 and $25 \mathrm{~h}$ of incubation along a temperature gradient $\left(15.6-32.1^{\circ} \mathrm{C}\right)$. Pico-eukaryotes from the East Australian Current (EAC) had relatively constant ROS and showed greatest mortality after $25 \mathrm{~h}$ at $7^{\circ} \mathrm{C}$ below ambient, whereas those from the Tasman Sea had elevated ROS in both warm and cool temperature extremes and greatest mortality at temperatures $6-10{ }^{\circ} \mathrm{C}$ above ambient, interpreted as the outcome of thermal stress. Tracking of water masses within an oceanographic circulation model showed populations had distinct thermal histories, with EAC pico-eukaryotes experiencing higher average temperatures for at least 1 week prior to sampling. While acclimatization and community assembly could both influence biological responses, this study clearly demonstrates that phenotypic divergence occurs along planktonic drift trajectories.
\end{abstract}

Studies of marine microbial responses to changing ocean environments have largely focussed on biogeographic shifts in community composition [1-3], or on detailed, short

These authors contributed equally: Allison Skinner McInnes, Olivier F. Laczka

Supplementary information The online version of this article (https:// doi.org/10.1038/s41396-019-0355-6) contains supplementary material, which is available to authorized users.

Martina A. Doblin

Martina.Doblin@uts.edu.au

1 Climate Change Cluster (C3), University of Technology Sydney, PO Box 123 Broadway, Ultimo, NSW 2007, Australia

2 QIMR Berghofer Medical Research Institute, 300 Herston Road, Herston, QLD 4006, Australia

3 School of Biological Sciences, University of Essex, Wivenhoe Park, Colchester CO4 3SQ, UK

4 Remote Sensing and Satellite Research Group, Curtin University, Building 301, Kent Street, Bentley 6102 WA, Australia

5 Department of Botany and the Beaty Biodiversity Centre, The University of British Columbia, Vancouver, BC V6T 1Z4, Canada (acclimation) and long (evolutionary) term responses of single strains in laboratory manipulations $[4,5]$. While insightful, these studies either omit examination of the physiological and ecological mechanisms that influence large-scale in situ population persistence, or remove microbes from their ecological context (e.g., investigate them in the absence of competitors or predators) in order to feasibly quantify detailed population-level responses. Observational, modelling, and experimental field studies that capture the legacy of past environmental exposure and

6 CSIRO Oceans and Atmosphere, 3-4 Castray Esplanade, Hobart, TAS 7004, Australia

7 Institute of Applied Ecology New Zealand, School of Science, Auckland University of Technology, Private Bag 92006, Auckland, New Zealand

8 W.K. Kellogg Biological Station, Michigan State University, 3700 E Gull Lake Drive, Hickory Corners, East Lansing, MI 49060, USA

9 Grantham Institute \& Department of Physics, Imperial College London, London, UK

10 Institute for Marine and Atmospheric Research Utrecht, Utrecht University, Utrecht, Netherlands 
the interactions between organisms [6] are therefore critical to understanding the processes regulating microbial population diversity and function in natural environments.

Here we use live cell probing and flow cytometry analyses at sea to examine the response of photosynthetic pico-eukaryotes within intact marine microbial communities (i.e., containing other phototrophs, bacteria, viruses, and grazers), to short-term temperature excursions. We used real-time underway sensing by means of thermosalinograph and acoustic doppler current profiler (ADCP) velocity to target microbial communities in different water masses. A rosette sampler (with attached Seabird SBE19 conductivity temperature and depth profiler) was used to capture surface seawater $(6 \mathrm{~m})$ from two sites, one in the EAC, a poleward-flowing western boundary current undergoing relatively rapid long-term warming compared to the global ocean $\left(0.8-1.8^{\circ} \mathrm{C}\right.$ per century, $2-3$ times the global average; [7]), and another in the adjacent Tasman Sea. Within $2 \mathrm{~h}$ of collection, microbial communities were incubated along a thermal gradient of six temperatures ranging from 15.6 to $32.1^{\circ} \mathrm{C}$, representing a departure from their ambient temperature $\left(\sim 22^{\circ} \mathrm{C}\right)$ of approximately -7 to $+10^{\circ} \mathrm{C}$. Using a 'dynamic phenotyping' approach $[8,9]$, we measured acute rather than acclimated thermal responses to gain insight about the potential for 'preconditioning' to high temperature in the pre-sampling period that would not necessarily be manifest under static conditions [9]. Such pre-exposure to high temperature can improve the thermal performance of photosynthetic eukaryotic microbes under heat stress [10].

The potential for temperature-induced stress was measured over a $25 \mathrm{~h}$ period by quantifying changes in the abundance of reactive oxygen species (ROS) positive cells (Fig. S1), and the size of the photosynthetic pico-eukaryote population. In photosynthetic organisms, the chloroplast, the mitochondrial electron-transport chain, and the peroxisome are the main sources of ROS [11]. Under physiological steady state, the ROS content of cells is controlled through enzymatic (e.g. glutathione peroxidase, catalase) and non-enzymatic scavenging processes (e.g. ascorbic acid; [12]). At low levels, ROS play an important role in pro-survival mechanisms, acting as signalling molecules involved in regulating development and pathogen defence responses, or as secondary messengers that transmit initial stress signals, allowing cells to react and adapt to different environmental cues [13, 14]. However, under stressful conditions (e.g. temperature extremes, UV exposure, pollution), ROS quantities overwhelm the capacity of antioxidants, allowing ROS to accumulate and potentially cause irreversible damage to proteins, DNA, and lipids, triggering programmed cell death processes such as apoptosis [15]. Thus, in our experiments, we hypothesised that exposure of populations to temperatures above and below ambient would cause an increase in cellular ROS (i.e., production would exceed scavenging), with potential asymmetry between warming and cooling because of the temperature dependence of enzymatic processes. Furthermore, the increase in ROS would potentially trigger a cascade leading to an increase in cell mortality-i.e., resulting in a decrease in the population relative to its initial abundance. The acute response to temperature excursions was captured by subsampling populations over a time-course $(0,1,5,25 \mathrm{~h})$, staining them with a commercially available kit that uses two stains to specifically target ROS produced in eukaryotic cells (superoxide, and all other ROS except superoxide; Enzo Life Sciences, Inc., New York, USA), and quantifying their fluorescence intensity (relative to a standard microsphere; see Supplementary Information, Fig. S1) by immediately analysing them with a flow cytometer (Influx Mariner, BD Biosciences).

Initially, there was a relatively low proportion of cells in the Tasman Sea versus EAC pico-eukaryote population that contained ROS above background levels $(10.9 \pm 2.7 \%$ compared to $44.0 \pm 3.2 \%$, respectively; mean $\pm \mathrm{SD}, n=3$; Fig. S4), with both populations showing effective upregulation of ROS production (Fig. S2) in the induced positive control $(92.1 \pm 0.8$ and $84.4 \pm 4.5 \%$ in the Tasman Sea and EAC, respectively). During the experiment, ROS expression in the EAC population remained relatively constant under upwards or downwards temperature excursions at all time points (Fig. 1a), suggesting scavenging processes were effectively maintained. In the Tasman Sea population, however, ROS fluorescence was immediately $(1 \mathrm{~h})$ higher at $+10{ }^{\circ} \mathrm{C}$ above ambient (ANOVA, $F=4.67, p=0.013$ ), with a relatively high proportion of stressed cells during the first $5 \mathrm{~h}$ of the assay (Fig. S4B). Furthermore, in contrast to the EAC, the time-course of ROS expression over $25 \mathrm{~h}$ showed a significantly different pattern amongst temperatures, with ROS fluorescence declining with time at high temperature (Fig. 1a, c; ANOVA temperature $x$ time interaction, $F=4.16, p=0.001$ ).

After $25 \mathrm{~h}$, the size of pico-eukaryote populations had changed significantly across temperatures (Fig. 1b). The Tasman Sea population declined by $80-90 \%$ at $32.1{ }^{\circ} \mathrm{C}$ but at other temperatures, the population was similar in size to the T0 samples (ANOVA, $F=31.19, p<0.001$ ). The EAC population, however, experienced $40-50 \%$ mortality across temperatures between 17.4 and $32.1^{\circ} \mathrm{C}$, with $\sim 90 \%$ loss of cells at the lowest temperature of $15.6{ }^{\circ} \mathrm{C}$ (Fig. 1b; ANOVA, $F=15.43, p<0.001)$. Overall, the Tasman Sea population appeared most sensitive to temperatures from +6 to $+10{ }^{\circ} \mathrm{C}$ above ambient, with the EAC population most sensitive to the coldest temperature $\left(-7^{\circ} \mathrm{C}\right.$ below ambient). There was a significant positive relationship between the amount of ROS fluorescence and the percentage of cells remaining in both the EAC and the Tasman Sea 

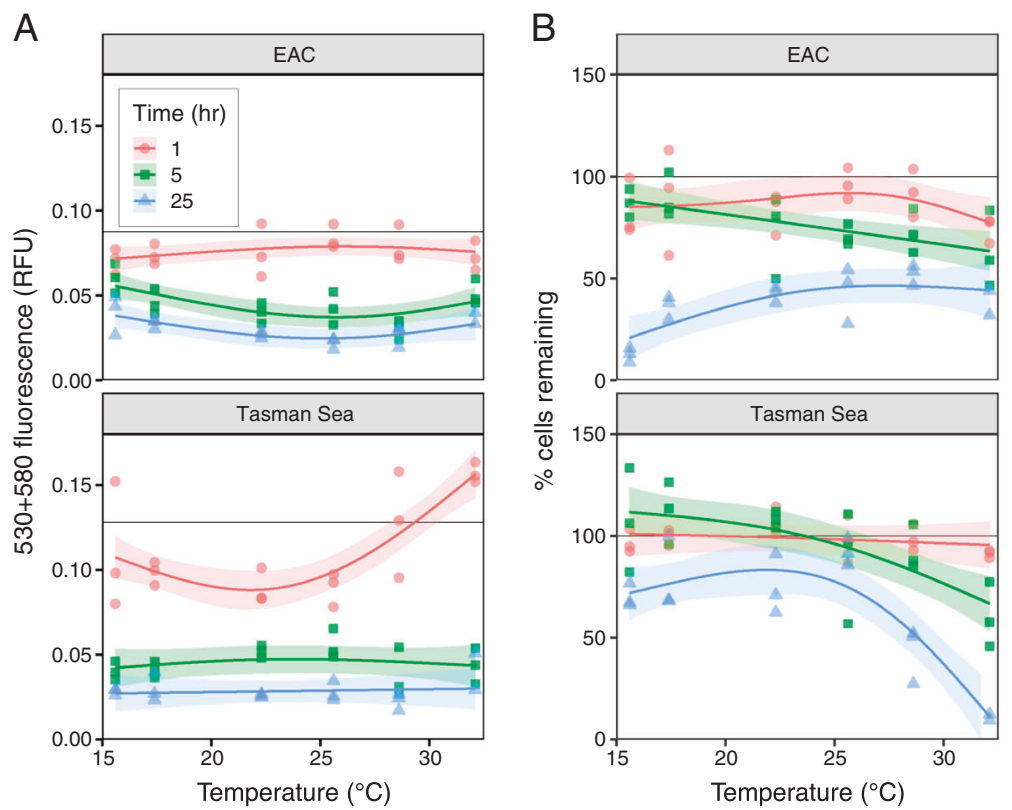

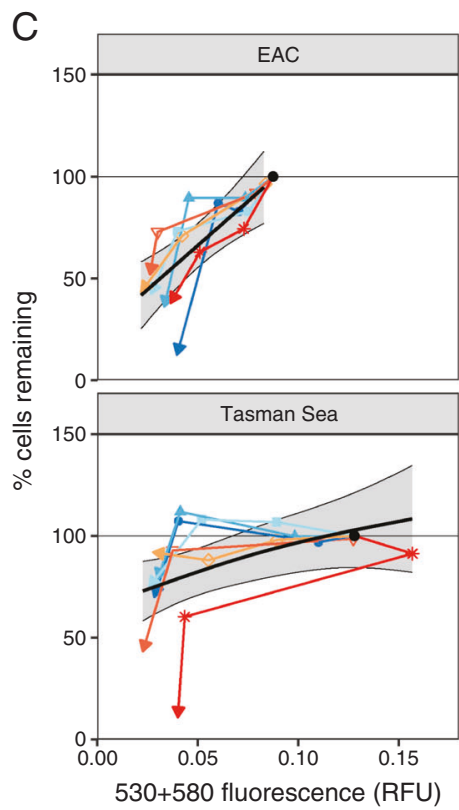

Temperature

$\left({ }^{\circ} \mathrm{C}\right)$

$\rightarrow 15.6$

$\simeq 17.4$

$-22.3$

$\neg 25.6$

$\nabla 28.6$

* 32.1
Fig. 1 Time-course $(1,5,25 \mathrm{~h})$ of the (a) bead-normalised reactive oxygen species (ROS) fluorescence $(530+580 \mathrm{~nm}$ relative fluorescence units, RFU) of cells in the EAC and Tasman Sea pico-eukaryote population at temperatures above and below ambient $\left(\sim 22^{\circ} \mathrm{C}\right)$ (Tables S1 and S2; Fig. S1); b Percentage of cells remaining in the EAC and Tasman Sea pico-eukaryote population after 1, 5 and $25 \mathrm{~h}$ of incubation at different temperatures (Tables S3 and S4). c The relationship between the percentage of pico-eukaryotes remaining and bead-normalised ROS fluorescence at $0,1,5$ and $25 \mathrm{~h}$ (arrows connect observations over time taken within the same water mass and temperature treatment, indicated by colours/symbols with a global average across all temperatures shown in black). Over time, in both water masses and across temperatures, the population size declined with decreasing bead-normalised fluorescence (Table S4 and S5); in the EAC it declines approximately linearly, but in the Tasman Sea the relationship is more curvilinear (Table S6) population (Fig. 1c; Generalised Additive Mixed Model, $p<0.001$ and $p=0.030$, respectively; Table S6).

It was apparent that temperature was not the only factor influencing cell survival during incubations-population dynamics within assays were likely mediated by the presence of other phototrophs, viruses, bacteria and grazers within experimental vessels. However, the dynamics of ROS within pico-eukaryotes was clearly influenced by temperature and revealed divergent physiology between water masses, suggesting that Tasman Sea cells experienced thermally-induced stress at the highest temperature which led to greatest mortality at $32.1{ }^{\circ} \mathrm{C}$ (Fig. 1c). In contrast, the EAC population appeared to maintain ROS scavenging processes across all temperatures (Fig. 1a).

We considered two possible explanations for the contrasting thermal responses of populations in the EAC and Tasman Sea: differences in the taxonomic composition of the phototrophic pico-eukaryote populations at the start of the incubation, and differences in their thermal acclimatization status. Amplicon sequencing of the chloroplast 16S rRNA gene from replicate samples at each site and subsequent bioinformatic analysis (PhytoREF, [16]) revealed that the two water masses shared $55 \%$ of photosynthetic microbial genera (Fig. S3; SIMPER, Primer v6; PRIMERE, Plymouth, UK). At higher taxonomic resolution (OTUs with $97 \%$ nucleotide identity) the two water masses shared
$17 \%$ of taxa, with greater diversity of OTUs in the Tasman Sea (Table S1). The taxonomic dissimilarity between the two water masses was primarily due to differences in the abundances of two chlorophyte OTUs: Bathycoccus (Mamiellales) and Prasinococcales, which comprised 15 and $1 \%$ (respectively) of EAC photosynthetic eukaryotes, and 5 and $8 \%$ of those in the Tasman Sea. Based on the known biogeography of Bathycoccus ecotypes, it is possible that the divergent pico-eukaryote thermal responses in the EAC and Tasman Sea were influenced by differences in the relative abundance of strains TOSAG39-1 and RCC1105, the latter of which prefers warmer water [17].

To assess the potential for differences in acclimatization between water masses, a high-resolution ocean circulation model with particle tracking software was used to estimate the thermal exposure of virtual microbes arriving to the sampling sites (Supplementary Information). Although surface seawater temperature at the time of sampling was similar (Table S1), microbial communities had distinct thermal histories, as shown by their different transport trajectories (Fig. 2a). Organisms sampled in the EAC likely originated from northerly locations and experienced higher average temperatures for several weeks (i.e., up to $\sim 20$ generations) prior to sampling, as compared to the Tasman Sea population (Fig. 2b). A sensitivity analysis was undertaken to assess how different the thermal history 

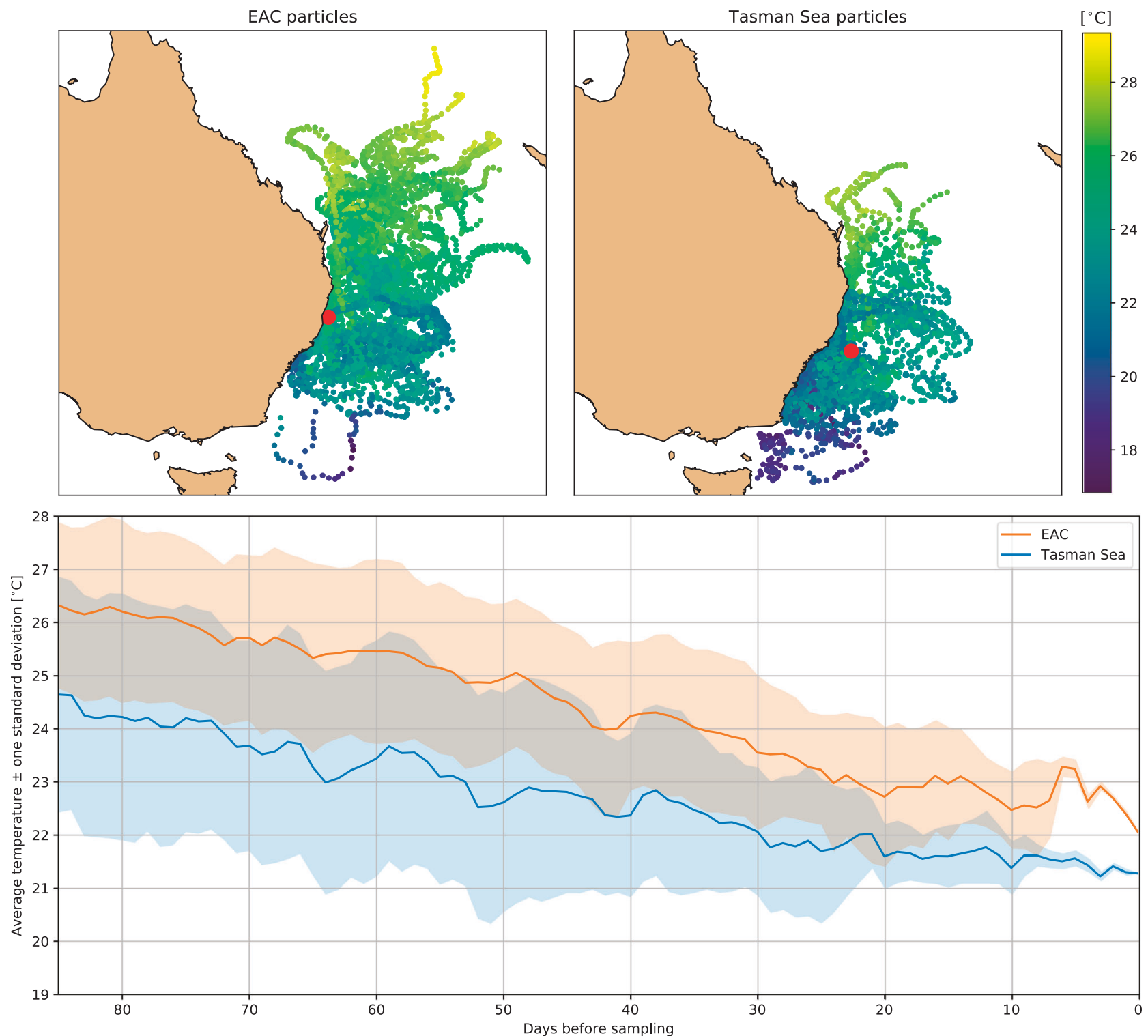

Fig. 2 Thermal exposure of virtual microbes arriving to the EAC (a) and Tasman Sea (b). A total of 100 virtual particles released at each oceanographic sampling site (red symbols) were backtracked for 85 days before the date of sampling (June 2015 austral winter),

would be if the populations were sampled up to 4 weeks prior/post the voyage (Fig. S5). The analysis shows that for six out of nine scenarios, temperature exposure 1 week prior to sampling (equivalent to numerous pico-eukaryote generations) is consistently different between the sampling locations, with the exception of 2-4 weeks after the voyage. This indicates that thermal exposure immediately prior to sampling is most likely to influence the physiology of picoeukaryotes observed in our study.

Although it is difficult to rank the importance of community assembly versus physiological acclimatization processes along drift trajectories, our data clearly demonstrate that advected populations diverge in their thermal recording the temperature of their locations. Panel c shows mean temperature (solid line) \pm one standard deviation (shaded area) experienced by particles from the EAC (orange) and Tasman Sea (blue)

performance, with pico-eukaryotes in a relatively warm western boundary current being less sensitive to high temperature-induced stress compared to those in adjacent waters. Furthermore, we determined that differences in ROS expression after $1 \mathrm{~h}$ of warming are indicative of mortality $24 \mathrm{~h}$ later, with largest increases in cellular ROS fluorescence corresponding to greatest population decline (Fig. S6). Differences in ROS production amongst picoeukaryotes may have been due to a direct effect of heat on the photosystems (a source of ROS; [18]), or an indirect effect via intermolecular interactions [19]. For example, prior exposure to relatively high but non-lethal temperatures could induce greater non-photochemical quenching 
(resulting in more effective heat dissipation), as has been demonstrated in symbiont zooxanthellae [10], or it could cause upregulation of heat shock proteins and molecular chaperones [20] that moderate impacts on enzymes and other proteins involved in ROS scavenging.

This study highlights the physiological differences in adjacent microbial populations, and suggests that the ability to tolerate high temperature may be an important trait influencing fitness and the capacity for range expansion amongst natural populations. While the exact mechanism remains to be elucidated, our results have clear implications for predicting the impacts of marine heat waves and longterm warming on ocean microbes. Further field experimentation will help bridge the gap between models and culture studies, revealing the limits of plasticity for microbes to dynamically respond to changing ocean conditions.

Acknowledgements The authors would like to thank the Marine National Facility for ship-time and technical support on the RV Investigator, as well as the captain, crew, and chief scientist, Prof Iain Suthers, for facilitating our sample collection during voyage IN2015_V03. This research was supported by an Australian Research Council Discovery Project (DP14010134) and Large Infrastructure and Equipment Fund (LE130100019) to MD. Molecular analyses were funded by an ARC Discovery Grant (DP140101045), awarded to Prof Justin Seymour; we thank him and Dr Nahshon Siboni for their support. CTK was supported by NSF grant OCE-1638958, MEL, KGB, JSC, CMR, LL and MA were supported by Australian Government Training Program Scholarships and OFL was funded by a University of Technology Sydney Chancellor's Postdoctoral Fellowship.

\section{Compliance with ethical standards}

Conflict of interest The authors declare that they have no conflict of interest.

Publisher's note: Springer Nature remains neutral with regard to jurisdictional claims in published maps and institutional affiliations.

\section{References}

1. Barton AD, Irwin AJ, Finkel ZV, Stock CA. Anthropogenic climate change drives shift and shuffle in North Atlantic phytoplankton communities. Proc Nat Acad Sci. 2016;113:2964-9.

2. Dutkiewicz S, Scott J, Follows MJ. Winners and losers: ecological and biogeochemical changes in a warming ocean. Glob Biogeochem Cycles. 2013;27:463-77.
3. Fuhrman JA, Cram JA, Needham DM. Marine microbial cymmunity dynamics and their ecological interpretation. Nat Microbiol. 2015;13:133-46.

4. Listmann L, LeRoch M, Schluter L, Thomas M, Reusch TBH. Swift thermal reaction norm evoluation in a key marine phytoplankton species. Evol Appl. 2016;9:1156-64.

5. Schulte PM, Healy TM, Fangue NA. Thermal performance curves, phenotypic plasticity, and the time scales of temperature exposure. Integr Comp Biol. 2011;51:691-702.

6. Doblin MA, van Sebille E. Drift in ocean currents impacts intergenerational microbial exposure to temperature. Proc Nat Acad Sci. 2016;113:5700-5.

7. Wu L, Cai W, Zhang W, Nakamura H, Timmermann A, Joyce T, et al. Enhanced warming over the global subtropical western boundary currents. Nat Clim Change. 2012;2:161-6.

8. Ruderman D. The emergence of dynamic phenotyping. Cell Biol Toxicol. 2017;33:507-9.

9. Cruz JA, Savage LJ, Zegarac R, Hall CC, Satoh-Cruz M, Davis $\mathrm{GA}$, et al. Dynamic environmental photosynthetic imaging reveals emergent phenotypes. Cell Syst. 2016;2:365-77.

10. Middlebrook R, Hoegh-Guldberg O, Leggat W (2008). The effect of thermal history on the susceptibility of reef-building corals to thermal stress. J Exp Biology 211:1050-56.

11. Apel K, Hirt H. Reactive oxygen species: metabolism, oxidativestress, and signal transduction. Ann Rev Plant Biol. 2004;55:373-99.

12. Gill SS, Tuyeja N. Reactive oxygen species and antioxidant machinery in abiotic stress tolerance in crop plants. Plant Physiol Biochem. 2010;48:909-30.

13. Mittler R, Vanderauwera S, Suzuki N, Miller G, Tognetti VB, Vandepoele K, et al. ROS signaling: the new wave? Trends Plant Sci. 2011;16:300-9.

14. Asada K. Production and scavenging of reactive oxygen species in chloroplasts and their functions. Plant Physiol. 2006;141:391-6.

15. Perez-Perez ME, Lemaire SD, Crespo JL. Reactive oxygen species and autophagy in plants and algae. Plant Physiol. 2012;160:156-64.

16. Decelle J, Romac S, Stern RF, Bendif EM, Zingone A, Audic S, et al. PhytoREF: a reference database of the plastidial 16S rRNA gene of photosynthetic eukaryotes with curated taxonomy. Mol Ecol Res. 2015;15:1435-45.

17. Vannier T, Leconte J, Seeleuthner Y, Mondy S, Pelletier E, Aury JM. et al. Survey of the green picoalga Bathycoccus genomes in the global ocean. Nature Sci Rep. 2016;6:37900

18. Pospísil P. Production of reactive oxygen species by photosystem II. Biochim Et Biophys Acta. 2009;1787:1151-60.

19. Feder ME, Hoffman GE. Heat shock proteins, molecular chaperones, and the stress response. Ann Rev Physiol. 1999;61:243-82.

20. Henkel SK, Hoffman GE. Differing patterns of hsp70 gene expression in invasive and native kelp species: evidence for acclimation-induced variation. J Appl Phycol. 2008;20:915-24. 